Fecha de recepción: diciembre 2017 Fecha de aceptación: octubre 2018 Versión final: julio 2019

\section{Aprendiendo a interpretar música por medio del Smartphone: la explicitación y reconstrucción de las representaciones encarnadas}

Juan Ignacio Pozo *, José Antonio Torrado ${ }^{\star 2}$ y

María Puy Pérez-Echeverría *3

\begin{abstract}
Resumen: En este artículo se expone un trabajo descriptivo de enseñanza de la música, basado en un caso, en el que, desde un enfoque emocional y encarnado, alejado de los métodos tradicionales, analíticos y basados en la partitura, se muestra el papel que juegan diferentes representaciones, desde las más encarnadas (la posición corporal, los gestos) hasta las más simbólicas (la partitura), en el proceso de redescripción representacional para dar sentido emocional a la música. Además, se estudia cómo el uso del Smartphone y otros dispositivos similares pueden ayudar a un acercamiento epistémico a la enseñanza y el aprendizaje de la música. Se discuten los diferentes enfoques de acercamiento a la música y las relaciones entre diferentes representaciones.
\end{abstract}

Palabras clave: emociones - representaciones encarnadas - representaciones simbólicas aprendizaje de la música - redescripción representacional.

[Resúmenes en inglés y portugués en la página 260]

(*) Juan Ignacio Pozo es Doctor en Psicología por la Universidad Autónoma de Madrid, en cuya Facultad de Psicología es Catedrático de Psicología Básica. Sus investigaciones están centradas en el aprendizaje en diferentes dominios de conocimiento, incluida la música. nacho.pozo@uam.es

${ }^{\left({ }^{*}\right)}$ José Antonio Torrado es Doctor en Historia y Ciencias de la Música y Master en Gestión y Promoción de la Música por la Universidad Autónoma de Madrid. Actualmente es catedrático de Violín en el Conservatorio Superior de Música del Principado de Asturias. joseantonio.torrado@gmail.com

(*3) María Puy Pérez-Echeverría es Doctora en Psicología por la Universidad Autónoma de Madrid, en cuya Facultad de Psicología es Profesora Titular de Psicología Básica. Sus investigaciones están centradas en el aprendizaje en diferentes dominios de conocimiento, incluida la música.mdelpuy.perez@uam.es 


\section{Introducción: la función de los códigos en la enseñanza tradicional de la música}

En la música, como en otros muchos dominios, los códigos y sistemas externos de representación desempeñan una función esencial en el proceso de producción y también en la enseñanza y aprendizaje. La educación musical, especialmente la clásica, ha partido de la necesidad del dominio de estos códigos. Más concretamente, la formación instrumental ha asumido que el aprendizaje del código sustentaba el resto de los aprendizajes y, específicamente, el dominio del instrumento. Igual que sucede en la escritura, las matemáticas o la ciencia, se ha tendido a instruir a los estudiantes en el dominio del código (la lectura de partituras), de forma tal que lo aprenden como un lenguaje abstracto, amodal y arbitrario (AAA) (Glenberg, de Vega y Graesser, 2008), descontextualizado de la propia acción, la interpretación, y de los significados que cobra en contextos concretos. Así, una vez iniciado el aprendizaje básico sobre cómo producir sonidos con el instrumento, se continúa con la decodificación del lenguaje musical escrito, para traducirlo a patrones sonoros que se deben ejecutar mediante acciones concretas en el instrumento. Tocar música se concibe como un proceso de adquisición de las destrezas técnicas necesarias para dominar un instrumento que permite traducir la partitura a otro código, a un sonido organizado, que es lo que finalmente conocemos como música.

Aunque hay muchas formas de enseñar el lenguaje musical y su traducción a patrones sonoros, los estudios muestran una concepción dominante en nuestra cultura, según la cual la música está contenida en la partitura. Tocar es traducir a sonidos lo que está escrito en una partitura y, por tanto, para poder tocar cualquier instrumento hay que saber decodificarla. En ese proceso de alfabetización musical se suele asumir además un enfoque analítico, en el que el texto musical se descompone en sus unidades mínimas, las notas, cuya posterior combinación o secuenciación acaba por producir la melodía deseada. De acuerdo con el modelo AAA mencionado (Pozo, 2017), la partitura se descompone en una serie de unidades abstractas, amodales y arbitrarias (las notas), de cuya combinación debe surgir el significado, de modo similar a cómo se propone a los niños el aprendizaje de la lectoescritura o la notación matemática. En el aprendizaje de la música, como en esos otros dominios, parece predominar un patrón cultural acorde con un enfoque asociativo (Pozo, 2008), según el cual el todo se reduce a la suma de las partes. Así, el significado, que, como veremos, en el caso de la enseñanza de la música remite a su contenido emocional (Kivy, 2001), surge de la asociación de las unidades más elementales que componen el código, aparece agregando notas.

\section{Aprendiendo a interpretar las partituras}

La investigación ha mostrado que esta forma de aprender a decodificar las obras musicales tiene importantes consecuencias. Así, diferentes estudios indican que los alumnos tienden a procesar las partituras a un nivel superficial, sobre todo en las primeras etapas de su aprendizaje. A partir de la distinción establecida por Postigo y Pozo (2004) sobre niveles de procesamiento de la información gráfica (ver también Pérez Echeverría, Postigo 
y Marín, 2018), Bautista et al (2009), Casas y Pozo (2008), López-Iñiguez y Pozo (2014) o Marín, Pérez Echeverría y Hallam, (2012) distinguen varios niveles de decodificación de las partituras, de complejidad creciente, que irían desde un procesamiento notacional, centrado en los elementos explícitos en la partitura (clave, tempo, notas, etc.), a un nivel o niveles intermedios, basados en un análisis sintáctico, que establece relaciones entre esos elementos mediante conceptos o estructuras (melodía, armonía, fraseo o textura., etc.) y, finalmente, un último nivel, expresivo o artístico, que desvela los aspectos expresivos, estilísticos, el significado musical e histórico de esa obra en relación con otras obras y con las intenciones expresivas del autor.

Igual que los lectores poco competentes suelen intentar aprender los textos literalmente, palabra a palabra, los datos muestran un procesamiento analítico ("nota a nota") en los aprendices de música. Solo en los niveles más avanzados tiende a aparecer un procesamiento más complejo, sintáctico o expresivo (Bautista et al., 2009, Marín, Pérez Echeverría y Hallam, 2012) que es el que permite dar verdadero significado musical a la obra. El problema del significado ha ocupado a los musicólogos a lo largo de la historia. Dado que en su mayor parte la música, a diferencia del lenguaje natural, carece de un objeto referencial externo, se ha tendido a buscar su significado en su estructura formal interna, en la forma en que internamente se organizan los sonidos (Davies, 1994). Sin embargo, en tiempos recientes parece haber un consenso en que el verdadero significado de la música reside en la respuesta que provoca en quien la escucha (Koopman y Davies, 2011), que nos remite por tanto a las emociones que comunica o elicita en el oyente.

Este procesamiento "nota a nota" de las partituras, que elude el problema del significado musical en favor de la mera reproducción de la estructura de sonidos, común en los niveles iniciales, pero también muy frecuente en los superiores, tiene la consecuencia de que la propia interpretación se concibe como la traducción literal de las notas a sonidos. Se establece así una correspondencia entre notas y sonidos, de forma que interpretar es reproducir la música que está explícita en la partitura, a través de un dominio técnico muy sofisticado del instrumento, que se convierte en el principal objetivo de la enseñanza instrumental. Se trata por tanto de trabajar la adquisición de secuencias motoras complejas, específicas para cada instrumento, que permiten generar el sonido deseado, canónico. Esta concepción considera que el logro del dominio técnico provocará a su vez una interpretación musical expresiva y sensible, que amplifique el contenido expresivo de la música o incluso influya emocionalmente en el oyente, que es la meta final de cualquier formación instrumental. La expresividad, por tanto, debe surgir motu propio de la propia técnica o bien de ciertas cualidades intangibles del intérprete que, por su propia naturaleza, no pueden ser enseñadas. De la misma forma que en la enseñanza de la lectoescritura o de la notación matemática se asume que el dominio técnico o algorítmico del código asegura su uso comunicativo o significativo, también parece asumirse que una correcta decodificación de las partituras y su traducción a acciones motoras asegurará la comunicación emocional a la que está dirigida la música (Juslin y Västfjäll, 2008; Koelsch, 2014).

Como consecuencia, la expresión de emociones desde la gestión del sonido ocupa un lugar muy secundario en las prácticas de enseñanza y aprendizaje instrumental. Cuando se analizan las concepciones sobre la enseñanza y el aprendizaje de profesores de música instrumental (Bautista, Pérez Echeverría y Pozo, 2010) o las de los alumnos (Marín, Scheuer y 
Pérez-Echeverría, 2013) se encuentran solo vagas referencias a que el objetivo de la música es "trasmitir emociones". Además, entre las actividades de enseñanza o las estrategias de aprendizaje no aparece apenas nada que facilite la gestión de las emociones (Torrado, Jiménez, Rucandio y Pozo, 2017). No es extraño por tanto que la emoción de la que más informan los intérpretes musicales de formación académica o clásica, es la ansiedad o el miedo escénico (Perdomo-Guevara, 2014), mientras que en otras culturas musicales como el flamenco o la música moderna, fruto de otros espacios educativos, se observa una gestión más significativa de las emociones en el proceso del aprendizaje instrumental (Casas-Mas, Pozo y Scheuer, 2015).

En resumen, desde esta perspectiva, los contenidos de aprendizaje musical y las metas de su enseñanza se restringen a la producción sonora (incluida la conversión a sonidos de la partitura) y al dominio de las demandas de producción del instrumento. Las metas y las acciones relativas a la naturaleza de la música, como la expresión o comunicación emocional, y del propio aprendizaje referido al cuerpo quedan reducidos a una mención marginal en las clases (Torrado et al, 2017). En nuestra opinión, esta negación de las emociones en el proceso de aprendizaje musical está relacionada con la ocultación del cuerpo como sistema de representación y gestión del propio aprendizaje en nuestra cultura educativa (Claxton, 2015; Pozo, 2017) y se manifiesta claramente en el lenguaje empleado en las clases de instrumentos de cuerda, en las que, por ejemplo, se habla de la punta del arco o del talón, pero no se menciona apenas el cuerpo como gestor del sonido ni como vehículo de las emociones. El predominio de una educación basada en el dominio de códigos AAA (abstractos, amodales y arbitrarios) se asienta en un dualismo profundamente arraigado en nuestra cultura, no solo en su dimensión educativa. Así, la educación debe cultivar la mente/alma del alumno en detrimento del cuerpo y, en definitiva, de la acción, el contexto y la implicación emocional (Pozo, 2017).

Tanto en las aulas y en el currículo, como en los laboratorios y en los desarrollos teóricos que analizan las formas de aprender y enseñar, ha predominado ese enfoque aséptico AAA (Glenberg, de Vega y Graesser, 2008; Pozo, 2017) que subyace a la forma en que se han desarrollado los procesos de alfabetización durante las últimas décadas, incluida la educación musical, en la que tan importante debería ser la función del cuerpo, como vehículo de las acciones que deben desarrollarse y sobre todo como sistema desde el que representar, sentir y reconstruir la propia actividad cognitiva, motora y emocional que hace posible la interpretación musical. Un nuevo enfoque educativo para la enseñanza instrumental requiere, por tanto, reconsiderar la función del cuerpo y su relación con los diferentes códigos y sistemas de representación externa que permiten regular su actividad al producir música.

\section{Un nuevo enfoque de la enseñanza de la música instrumental}

Recientemente, el enfoque AAA, que subyace a los procesos de alfabetización, está siendo fuertemente contestado desde la filosofía de la mente (Rowlands, 2010), la neurociencia (Damasio, 1994) y la psicología cognitiva y del aprendizaje (Claxton, 2015; de Vega, Glenberg y Graesser, 2008; Pozo, 2001, 2014; 2017, Wilson, 2002), y también desde los propios 
espacios educativos (Barab y Dodge, 2008). Se propone así un nuevo enfoque, basado en la llamada embodied mind (que traduciremos por mente encarnada), que define nuestra mente como un sistema encarnado, incorporado. Frente a la disociación mente-cuerpo y la creencia de que todo el conocimiento se inicia como una actividad abstracta, formal, descontextualizada, se parte de que toda actividad mental se produce desde y para el cuerpo. Es una actividad encarnada. Frente al supuesto evangélico de que "en el principio era el Verbo" que luego "se hace Carne", sobre el que se asienta buena parte de nuestra cultura, el enfoque encarnado asume que todos nuestros actos de conocimiento comienzan implícitamente en el propio cuerpo, en las sensaciones y representaciones corporales o encarnadas. Por tanto, conocer es en buena medida explicitar y reconstruir esas representaciones encarnadas primarias (Pozo, 2001, 2014). A diferencia de lo que sucede en el enfoque cognitivo clásico del procesamiento de información, en el que esas representaciones se codifican en forma de unidades simbólicas abstractas, amodales y arbitrarias) (de Vega, Glenberg y Graesser, 2008) desde el enfoque encarnado se asume que las representaciones primordiales tienen un carácter enactivo, se basan en acciones y sensaciones corporales, por lo que constituyen verdaderas represent/acciones (Pozo, 2014).

De esta forma, la enseñanza y el aprendizaje, incluido el aprendizaje musical (Cox, 2016), deberían dirigirse a explicitar y transformar esas representaciones encarnadas primarias por medio del dominio de nuevos códigos y sistemas de representación (Pozo, 2014, 2017). Todo aprendizaje debería partir de las representaciones implícitas y encarnadas, que inconscientemente guían la actividad de los aprendices. Pero, como veremos, esta transformación requiere su recodificación mediante diferentes lenguajes y representaciones externas de carácter social o cultural (Pérez Echeverría, Martí y Pozo, 2010; Pérez Echeverría y Scheuer, 2009), que no solo ayuden a explicitarlas sino sobre todo a generar nuevos significados mediante una redescripción representacional (Karmiloff-Smith, 1992) en otros formatos representacionales. El proceso de redescripción, de acuerdo con Karmiloff-Smith (1992), implicaría la traducción progresiva de una representación a un nuevo código o formato, con mayor potencia representacional. En el caso de la música instrumental estaría indicando cómo las representaciones intuitivas, ligadas al cuerpo, adquieren un significado diferente al explicitarse y traducirse a otros códigos externos, hasta adquirir un carácter más simbólico y más flexible por medio de las partituras. Dado que las representaciones encarnadas están encajadas o ligadas al contexto, se necesita traducirlas a otros códigos con más capacidad de transferencia que las propias experiencias corporales, para que lo aprendido pueda generalizarse a otros contextos o situaciones.

En el caso de la música instrumental, frente al enfoque tradicional AAA previamente descrito, se propone un nuevo enfoque, basado en estos supuestos de la mente encarnada (Torrado, Pozo y Braga, 2016). Como muestra la Figura 1, el modelo distingue cuatro componentes básicos o, si se prefiere, cuatro dispositivos representacionales que es necesario considerar para esta nueva forma de educación musical:

- Las emociones que se pretenden comunicar (el contenido emocional de la música que constituye su auténtico significado).

- Los sonidos y sus características, que permiten vehicular esas emociones. 
- Las propiedades y características técnicas del instrumento que restringen las acciones y que permiten generar esos sonidos.

- Las acciones y sensaciones corporales ligadas a la producción de esos sonidos con contenido emocional a través del instrumento.

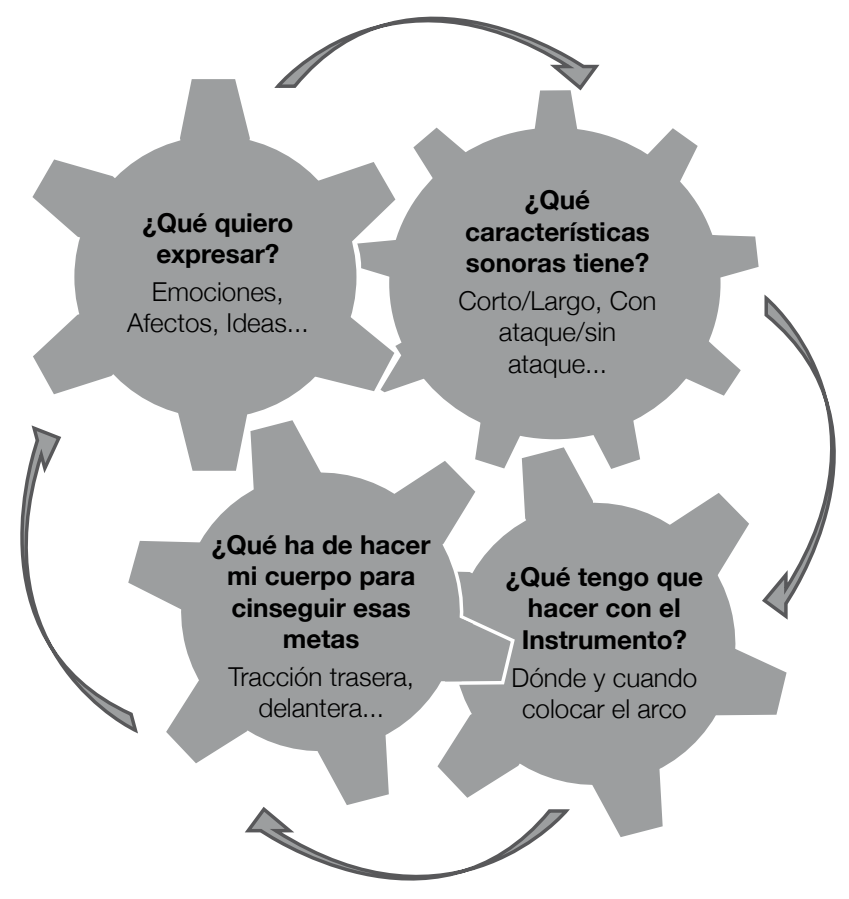

Figura 1. Componentes representacionales del aprendizaje instrumental

La representación emocional del contenido comunicativo, además de llevar implícita una respuesta corporal, debe modular el sonido que se quiere producir. Comunicar al oyente tranquilidad (pretender literalmente una respuesta corporal relajada), supone usar sonidos largos, con poco ataque, y poco material sonoro (Gabrielsson y Lindström, 2010). El diseño de producción sonora del instrumento nos indica qué cantidad de arco o aire usar, a qué velocidad, etc. A través de la representación mental de las propias acciones corporales se deben gestionar los movimientos que hagan posible la actividad demandada por el instrumento y así producir un sonido que reúna los parámetros que provoquen en el 
oyente esa respuesta emocional, de carácter también corporal. En la partitura sólo se explícita una blanca en dos por cuatro y sobre el pentagrama una anotación: "lento".

La traducción directa desde la partitura a un sonido preestablecido requiere recursos representacionales diferentes que los que se necesitan para usar el sonido con el fin de comunicar emociones. En el primer caso, no es necesario atender a las representaciones encarnadas, mientras que en el segundo es necesario partir de ellas y reconstruirlas para gestionar las acciones corporales sobre el instrumento que harán posible esa interpretación emocional.

Así, interpretar es algo más que decodificar y traducir la partitura a sonidos (Cook, 2013). Requiere establecer metas expresivas que deben ser reguladas y ejecutadas por medio del instrumento mediante la explicitación y el control metacognitivo de las propias acciones corporales (Torrado, Pozo y Braga, 2016). La música no está contenida en la partitura ni en el instrumento. Es el intérprete quien transmite las emociones a partir del control y regulación de su propio cuerpo y del sonido generado con sus acciones en un instrumento. Por tanto, el aprendizaje de la interpretación musical no requiere traducir directamente la música codificada en la partitura, sino:

1. Explicitar las metas expresivas deseadas, lo que a su vez, requiere

2. Establecer relaciones entre los diversos componentes representacionales (emoción, sonido, instrumento, cuerpo), lo que a su vez conlleva

3. Explicitar también los medios para lograrlas (regular las acciones y sensaciones corporales que deben desplegarse para producir los sonidos deseados).

Desde este modelo, esa explicitación solo se logrará mediante la mediación de diversos tipos de representaciones externas, en las que debe apoyarse la acción docente.

\section{Nuevos códigos para el aprendizaje de la música}

En el enfoque tradicional de la educación musical que hemos esbozado, el código en el que se representa la música es fundamentalmente la partitura. Sin embargo, desde este nuevo enfoque hay una variedad o pluralidad de lenguajes o tipos de representación que pueden emplearse para traducir o redescribir unas representaciones en otras. Algunos de esos códigos, por ejemplo la mímesis, se hallan más cercanos a las representaciones encarnadas, mientras que otros, como las partituras, por su naturaleza simbólica, tienen un carácter más arbitrario (o menos motivado) (Martí, 2003). Comenzando por los códigos más motivados o más cercanos a la acción corporal hacia los más abstractos, podemos distinguir los siguientes tipos de representaciones externas (ver Pozo, 2017).

\section{Las acciones corporales como representaciones externas}

Según el modelo de las representaciones encarnadas, gran parte de nuestro conocimiento se codifica por medio de acciones corporales, aprendemos por medio del cuerpo y en el 
cuerpo. De hecho, nuestras acciones corporales comunican nuestras representaciones de forma implícita, tanto para quien expresa las representaciones como para quien las recibe. Pero también pueden usarse de forma deliberada o intencional en la enseñanza como vehículos para explicitar las representaciones encarnadas o incluso como recurso para adquirir nuevas representaciones explicitas. En este sentido, las acciones corporales pueden utilizarse como punto de partida para la redescripción representacional, a través de su recodificación en otros sistemas representacionales más potentes y descontextualizados. El estudiante que está aprendiendo a tocar el violín puede tomar conciencia de sus representaciones corporales si se graba mientras toca (su tensión corporal, la relación entre el movimiento del arco y el sonido obtenido, etc.), pero también puede observar a su maestro o a otro alumno tocando o incluso imaginarse a sí mismo tocando y experimentando esas sensaciones corporales. Se puede ayudar a aprender a tocar un instrumento musical usando el propio cuerpo como representación externa (Torrado, Pozo y Braga, 2016).

\section{El gesto como acción corporal suspendida}

Otro tipo de representación externa que nos permite reflexionar sobre nuestra propia práctica es el gesto o la mímesis, usado no solo como parte de las representaciones encarnadas que acompañan a la acción y pueden comunicar nuestras intenciones, deseos y emociones de forma implícita (gestos que acompañan al habla, la expresión facial de las emociones, etc.) sino también como un modo de comunicar deliberadamente o representar nuestras propias representaciones. En estos casos -por ejemplo, al emular el movimiento del brazo al mover el arco, pero sin arco ni violín- la acción manifiesta no se completa sobre el objeto, sino que se suspende, en el sentido que da Rivière (2003) a este término, según el cual quedan sin efecto las consecuencias materiales de esas acciones. Goldin-Meadow (2003) diferencia entre distintos tipos de gestos, según sus funciones comunicativas. Son especialmente interesantes para nuestros objetivos los gestos icónicos y los metafóricos. Los primeros representan "movimientos corporales, de objetos o personas en el espacio y formas de objetos o personas" (Goldin-Meadow, 2003, pág. 7), es decir, representan por analogía la forma de una acción o un objeto. Los gestos metafóricos no representan la forma de un objeto o una acción material, sino un concepto o una idea, y por tanto suponen ya una recodificación o redescripción representacional de una acción. Por ejemplo, se puede expresar la necesidad de aumentar la intensidad o la cantidad de sonido en una interpretación con un movimiento ascendente de la mano con la palma hacia arriba. A su vez, Kirsh (2013) identifica otro tipo de gesto plenamente explícito o deliberado, al que denomina "marking" y que consiste en esbozar una acción física sin realizarla completamente, es decir dejándola suspendida. El rasgo fundamental de estos esbozos es que no recrean la acción completa -en el caso de Kirsh (2013) una coreografía-, sino que realizar estos gestos de forma suspendida, sin completar la acción, tiene la meta clara de representarla o anticiparla, para así poder actuar cognitivamente sobre ella y regularla. 


\section{Los objetos como representaciones externas}

Buena parte de las acciones corporales están mediadas por objetos a través de los que vemos o sentimos nuestro cuerpo u otros objetos, y que nos permiten así explicitar algunas de sus propiedades representacionales. De esta forma, los objetos que manipulamos sirven como herramientas para transformar físicamente otros objetos, pero también como instrumentos mediadores en la acción simbólica. Transforman la mente de quien los usa, convirtiéndose en mediadores representacionales de las propias acciones (Vygotski, 1978). Este uso representacional o epistémico de los objetos los convierte en mediadores en los procesos de explicitación. Los propios instrumentos musicales son tanto herramientas para producir sonidos, como vehículos de algunas de las propiedades de ese sonido, de forma que explicitar sus características y sus funciones ayuda a redescribir el propio conocimiento musical encarnado. Además, pueden usarse esos u otros objetos (un metrónomo, la mano golpeando la mesa) para medir el tiempo o representar otras propiedades del sonido.

\section{El lenguaje hablado como explicitación de la acción}

Quizás el sistema más “natural” para redescribir nuestras representaciones sea el uso del habla o lenguaje oral. Tomasello (1999) destaca que el lenguaje es sobre todo un sistema para representar perspectivas con respecto a los objetos y el mundo en general. Las representaciones simbólicas tienen una mayor potencia representacional que la mímesis, ya que permiten una mayor descontextualización con respecto a su marco de referencia, al espacio y al tiempo (Donald, 1991). De hecho, como habla privada, con frecuencia acompaña a los intentos por aprender música (Casas-Mas, López-Î̃niguez, Pozo y Montero, 2018), de tal forma que se utiliza con una función epistémica para explicitar la acción y para comunicar a los demás y a uno mismo los obstáculos encontrados en el aprendizaje.

\section{Representaciones externas codificadas}

Aunque cada uno de estos tipos de representación externa ayuda a redescribir nuestras representaciones primarias, encarnadas y contextuales (Pozo, 2017), la plena descontextualización, y la posibilidad de relación con otras representaciones, no se alcanza hasta el uso de notaciones o registros que perduran más allá del momento de su producción. El uso de representaciones externas organizadas mediante códigos formales, convencionales, que producen representaciones permanentes permite que estas puedan a su vez convertirse en objeto de representación (Martí, 2003). Con ello se accede a una actividad mental teórica (Donald, 1991), con plena capacidad metacognitiva. La lectura y la escritura ayudan a no solo a transmitir ideas musicales, sino también a repensar las propias experiencias.

La incorporación de nuevas tecnologías permite el uso de otros soportes o lenguajes como los programas informáticos para la creación e interpretación musical, los DAW (Digital Audio Workstation) o apps. Por otro lado, pueden usarse los dispositivos móviles, como los 
Smartphone, para repensar o redescribir las propias experiencias musicales grabando los ensayos o las clases, de forma que se puedan acceder a las acciones corporales y los gestos e intercambiando mensajes que faciliten una reflexión sobre esas acciones y así ayuden a explicitarlas.

\section{Los sistemas externos de representación musical: la lectura de partituras}

Finalmente, aunque las partituras en este enfoque pierdan el papel central que ocupan en la educación musical tradicional, no por ello dejan de ser muy relevantes. No obstante, en esa tradición se fomenta un procesamiento superficial de las partituras, ligado a la transcripción directa a los sonidos. Aquí se trata de fomentar los niveles más complejos de procesamiento, vinculados a la definición de metas expresivas, que obviamente no están contenidas de modo explícito en la partitura, sino que forman parte la interpretación que se hace de la misma. Se ha comprobado que la interpretación de músicos relativamente expertos, a partir de una partitura, se vuelve mucho más compleja y autorregulada cuando están guiados por una meta expresiva (Torrado, Pozo y Braga, 2016). La partitura ya no es un registro del sonido que hay que producir sino una guía, que permite regular los diferentes componentes (cuerpo, sonido, instrumento y emoción) que conforman la interpretación musical.

El objetivo del presente trabajo es ilustrar, mediante un estudio de análisis de un caso, el uso de estos diferentes tipos de representaciones externas para la explicitación y la reconstrucción de las representaciones encarnadas en el aprendizaje de la interpretación musical. Se trata de un estudio descriptivo en el que, en el marco de clases de instrumento, se analizan los usos y funciones de diversas representaciones externas, sirviéndose para ello del Smartphone como herramienta para fomentar la reflexión de los alumnos sobre lo que hacen y las decisiones que toman. De entre los muchos recursos y sistemas representacionales que pueden usarse para favorecer los procesos de redescripción representacional, el Smartphone es un dispositivo que permite usar fácilmente diversas herramientas multimedia, incluidas algunas utilidades o aplicaciones con las que los alumnos están muy familiarizados y que pueden usarse con fines epistémicos para el aprendizaje, en este caso de la música. En concreto, los Smartphone, disponibles ya en todas las aulas, permiten no solo registrar imágenes y sonidos, sino almacenar e intercambiar con agilidad esos registros, que pueden además acompañarse, por ejemplo, en forma de WhatsApp, con mensajes basados en textos, emojis y otros códigos. Esta naturaleza multimedia -es decir al uso simultáneo y combinado de múltiples y variadas representaciones externas- unida a su familiaridad para los alumnos, puede hacer de los Smartphone una herramienta privilegiada para favorecer los procesos de explicitación del conocimiento en las aulas, al proporcionar, de forma fácil y rápida, tanto al profesor como al alumno diferentes tipos de representaciones externas, que median en los procesos de explicitación y ayudan a fomentar la autorregulación y el control metacognitivo de la interpretación musical. De manera más concreta, queremos observar cómo una estudiante de música gestiona su propio cuerpo o los diferentes tipos de representación con el objetivo de aprender a transmitir emociones mediante la música. 
Como puede verse, a medida que esos códigos se alejan más del soporte encarnado inicial, de las sensaciones corporales ligadas a la acción y la experiencia musical, van incorporando como contenido -en un sentido literal- las representaciones más primarias o más vinculadas al cuerpo. No es que unas representaciones sustituyen a otras sino que las integran o, si se prefiere, las redescriben, ya que aquellas más simbólicas tienen un mayor potencia representacional, mayor capacidad de abstracción o descontextualización y, por tanto, permiten traducir las representaciones codificadas en formatos más ligados al propio cuerpo.

\section{Estudio empírico}

\section{Metodología}

\section{Participantes}

En este estudio se analizará una clase de un profesor con una alumna principiante en un conservatorio. El profesor (el segundo de los autores de este artículo), tenía 30 años de experiencia docente en conservatorios profesionales y superiores, experiencia investigadora sobre aprendizaje musical y una trayectoria asentada como violinista. Sus concepciones y métodos de enseñanza se alejan de los modelos tradicionales, de acuerdo con el modelo antes presentado.

La estudiante a la que llamaremos Lara, de 10 años de edad, recibía clases individuales de una hora de duración, una vez por semana. Cursaba cuarto grado de formación de violín. Por tanto, tenía una experiencia previa de tres años de clase, cada uno con un profesor diferente, pero todos ellos con una metodología clásica, dirigida a la decodificación de sonidos. Durante el curso que se describe ha cambiado de profesor y de métodos de enseñanza-aprendizaje.

\section{Las clases de música instrumental}

La estrategia didáctica se basaba en el modelo antes descrito (en el apartado "un nuevo enfoque de la enseñanza de la música instrumental”). Después de utilizar estrategias dirigidas a que la alumna encuentre un sentido de la partitura, su percepción primaria sobre qué expresar, su meta era ayudar a la estudiante a que usara los sonidos de forma estratégica para impactar en las emociones del oyente, con un control y gestión metacognitivos del propio cuerpo que permitiera activar los mecanismos instrumentales productores de los sonidos (ver Figura 1). Las clases comenzaban con una reflexión sobre las tareas realizadas por la estudiante en casa desde la clase anterior. Después se realizaban varias actividades, organizadas siempre en forma de diálogo. Algunas actividades eran grabadas por el profesor en videos cortos por medio del Smartphone. Una vez finalizada la actividad, ambos dialogaban sobre el logro alcanzado en función de las metas y de la gestión de 
los diferentes componentes del aprendizaje instrumental (cuerpo, sonidos, instrumento y emociones expresadas). En las sesiones de estudio en casa, en ausencia del profesor, se pedía a la estudiante que grabara pasajes de su interpretación, los analizara y valorara en pequeños textos que enviaba al profesor por WhatsApp junto con el pasaje grabado. Esos mensajes enviados por WhatsApp servían como material para el inicio dialogado de la clase siguiente.

Aunque el uso del Smartphone tenía también otros objetivos didácticos, en este estudio mostraremos solo los diferentes tipos de representaciones externas utilizadas (la observación de las propias acciones corporales, el uso de gestos y representaciones miméticas, de objetos como mediadores y de diferentes códigos de representación musical, incluidas las partituras) y cómo éstas colaboran en la construcción estratégica del contenido emotivo, especialmente en fomentar la regulación metacognitiva por parte de la alumna.

\section{Recogida y análisis de datos}

Se grabaron en total 14 clases, casi la mitad de las clases impartidas, mediante una cámara fija situada al fondo del aula con el fin de producir la menor interferencia posible. Para este estudio se ha seleccionado una clase, situada a mitad de curso, concretamente la séptima grabación en la semana 12 del curso. Además, se han analizado las grabaciones y correos enviados por la alumna a su profesor durante los meses siguientes. Los representantes legales de la estudiante proporcionaron los permisos necesarios para realizar las grabaciones en el aula y para usar tanto el texto como las imágenes de los WhatsApp en el contexto de esta investigación.

Los fragmentos seleccionados fueron analizados por los tres investigadores, buscando episodios que explicitaran de qué forma las representaciones externas pueden servir para las tres funciones epistémicas antes enunciadas: (a) la reflexión sobre las metas de las actividades; (b) la relación entre los componentes de la actividad instrumental (cuerpo, instrumento, sonido y emoción); y (c) la regulación metacognitiva de la alumna, en términos de planificación, supervisión y evaluación de sus acciones.

De acuerdo con el carácter descriptivo o cualitativo del presente estudio, se presentarán algunos de los episodios seleccionados, analizando cómo el uso de las representaciones externas sirve para cada una de las tres funciones mencionadas: negociación de las metas, establecimiento de relaciones entre los componentes del aprendizaje instrumental y regulación metacognitiva de la propia actividad instrumental. Para ello se presentarán esos episodios, describiendo las acciones que se llevan a cabo y recogiendo las verbalizaciones de la estudiante y del profesor que ayudan a dar sentido a los procesos que están mediando en su aprendizaje. También analizaremos algunos de los mensajes y videos mandados para presentar el trabajo en casa. 


\section{Resultados}

\section{Metas del aprendizaje instrumental}

La clase de Lara (L) comienza con el siguiente diálogo con su profesor (P):

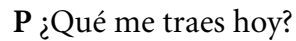

L Era (...) se llamaba el allegro de... ¿o era la escala?

P Sí, pero ¿qué voy a ver? ¿la escala?

$\mathbf{L}$ (asintiendo con la cabeza) $\mathrm{Mmm}$

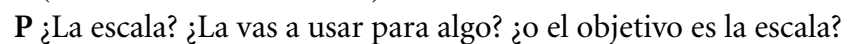

$\mathbf{L}$ (musitando) Mmmm... algo

$\mathbf{P}$ Bueno, pues cuéntame para qué es ese algo

L ... Mmm pues... Para que la gente se ponga contenta

$\mathbf{P}$ iiipara que la gente se ponga contenta!!! ¿y qué vas a hacer para conseguirlo?

L ....Pues... no sé.....con......con....iiiialegría?!!!

P Muy bien .... . ... ¿cómo vas a sacar esa alegría? ...por el sonido

En este comienzo, representativo de la estrategia didáctica del profesor, frente a modelos más tradicionales en los que se impone la voz del docente (López-Iñiguez y Pozo, 2016; Torrado y Pozo, 2008), puede verse que las intervenciones docentes adoptan la forma de preguntas y sugerencias, que, en el marco de una interacción dialógica, ayudan a la alumna a explicitar sus metas y sobre todo a redescribirlas o reformularlas en un código simbólico con mayor fuerza representacional. De hecho, se puede ver la diferencia en las metas de la estudiante y las que busca trabajar el docente y cómo este intenta transformar esas metas iniciales y el foco atencional de la alumna a través de un proceso de explicitación o reflexión conjunta basada en el diálogo. La estudiante quiere mostrar los resultados que ha logrado tras los ensayos en casa ("el allegro...la escala"), mientras que el profesor intenta orientarle hacia las metas expresivas de su interpretación (“¿la vas a usar para algo?”), lo que, como veremos, le lleva a establecer relaciones entre esas metas, el sonido, real o imaginado y la propia acción corporal sobre el instrumento.

Estas metas más reproductivas de Lara se observan claramente también en los videos y mensajes que envía mediante su Smartphone tras la clase, aunque en estos casos la reproducción, tras la intervención del docente, se centra en los movimientos corporales:

WhatsApp $2^{1}$ : En el primer video que te mandé la colocación de los dedos que me enseñaste en una parte no me acuerdo. Perdón

WhatsApp 1: Yo creo que ahora me sale bien porque he mirado la partitura y he colocado los dedos bien y también he hecho muy bien lo de los cambios de posición eso creo. //: Que crees tú?

La alumna ya no está tan preocupada en qué toca sino en cómo lo toca. Fija sus metas en sus propias acciones sobre el instrumento, con una orientación claramente técnica, observando cómo coloca los dedos y los cambios de posición en relación con el instrumento. En esta reformulación de las metas está mediando sin duda el que la alumna haya observado 
la grabación de sus acciones, de tal modo que se convierten en representaciones externas mediante las que pueden recodificarse o redescribirse las propias metas del aprendizaje. También el uso de códigos externos (mensajes de WhatsApp) facilita ese proceso de explicitación y transformación de las metas.

Pero, los cambios que busca el profesor mediante esos diálogos y reflexiones en el aula son más profundos, ya que quiere orientar las metas hacia el propio cuerpo y la expresión de emociones, como meta de la actividad instrumental:

$\mathbf{P}$ Ahora con el violín. Coge el violín pero no lo toques. Concéntrate en tus - movimientos. Ya sonará el violín. ¿Nos entendemos? Recuerda que el violín es un conversor o como le llamemos... lo importante es lo que le trasmitimos

$\mathbf{P}$ (Tras afinar el violín) Recuerda que ahora le damos al cuerpo. Ya sonará el violín (Lara toma el violín y el arco, se coloca, cierra los ojos y empieza a tocar la escala muy concentrada)

P Espalda extrema (va señalando con el dedo, afirmando y guiando los movimientos mientras Lara toca) Es ella, es ella la que toca. No es el violín es... es Lara la que toca...

De hecho, con el tiempo, las metas de Lara, parecen ir evolucionando poco a poco. En sus evaluaciones de los videos enviados a través de WhatsApp, además de referirse a las acciones sobre el instrumento, empieza a definir nuevas metas, relacionadas con sus propias sensaciones corporales mientras toca o con las emociones que intenta expresar:

WhatsApp 8: he mejorado en lo que hacía mal. //Aumentar el sonido y expresividad y mantener la concentración en todas las notas que salen de mi cabeza.

WhatsApp 9: Creo que lo he hecho bien porque en esta obra al principio estaba sintiendo emoción y después me iba cambiando hasta el: RE l la fa la re la la fa re...oos tresillos

En este fragmento, Lara establece metas mucho más ricas, que relacionan entre sí diferentes componentes (emoción, sonido, cuerpo) con la mediación de diferentes representaciones externas (la partitura, la observación de sus propias acciones en el video, la escritura mediante WhatsApp, o el uso de otros códigos como los emojis). Ya no está interesada en qué está tocando (allegro) o en las exigencias técnicas de su interpretación (cambios de posición), sino que está "concentrada" en lo que siente cuando toca y en lo que quiere tocar y sentir. Pero para poder relacionar esas metas con sus acciones y sensaciones necesita esos códigos que facilitan la redescripción representacional de sus sensaciones y acciones encarnadas.

\section{Relaciones significativas entre los componentes}

Este cambio en las metas se relaciona con la explicitación de relaciones significativas entre los diferentes componentes representacionales de la interpretación musical (emoción, cuerpo, instrumento, sonido) que ayudan a redescribir sus propias representaciones iniciales. Dicha explicitación y relación puede observarse en los diálogos mantenidos por el 
profesor y la estudiante durante la clase, apoyados en el uso de diferentes representaciones externas. Si antes veíamos un diálogo en el que se establecía como meta expresar alegría cuando se toca la escala, la siguiente conversación entre Lara y su profesor trata sobre los componentes que ayudan a expresar esa alegría:

P Muy bien .... . ... ¿cómo vas a sacar esa alegría? ...por el sonido

L ... Con las notas (al mismo tiempo hace como si cogiera un violín imaginario y tocara)

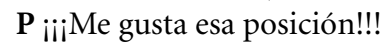

L Con las notas..... y así con....más. con... con ...con (simula el movimiento mediante gestos) $\mathbf{P}$ iiiPero si lo estás haciendo muy bien!!! Con las notas y ¿con más o menos ataque?

L Más ataque

$\mathbf{P}$ Venga vamos a ver todo esto

Para acercarse a su meta (la alegría), Lara es consciente de que debe gestionar los sonidos mediante ciertas acciones corporales (que explicita mediante una acción mimética, un tipo de gesto muy utilizado por el profesor durante las clases). De hecho, a continuación del diálogo, el profesor pide a Lara que se lo enseñe tocando "sin violín". Este recurso, tocar con un violín imaginario, es aquí un mediador esencial para explicitar las relaciones entre los movimientos corporales y la regulación del instrumento. Como hemos visto, este "hacer como" si se tocara el violín, suspendiendo la acción (Rivière, 2003), es un gesto de "marking" (Kirsh, 2010), o esbozo, en el que se utiliza el propio cuerpo y sus acciones como representación externa y ayuda a la toma de conciencia de los movimientos realizados, sin que el sonido o el instrumento puedan "desviar la atención”.

En el diálogo descrito antes, Lara, tras responder que va a usar las notas para mostrar alegría, responde haciendo varios gestos con el brazo que anticipan las respuestas verbales (notas cortas, con ataque), más dubitativas y emitidas solo gracias a las ayudas prestadas por el profesor, y que constituyen un ejemplo de la utilización del gesto icónico como medio de explicitación (Goldin-Meadow, 2013). Siguiendo a Goldin-Meadow, podríamos aventurar que estos gestos icónicos son en cierto modo la Zona de Desarrollo Próximo de Lara. Es capaz de explicitar por sí sola mediante gestos lo que sólo más tarde puede explicitar verbalmente gracias al andamiaje proporcionado por su profesor. La mediación docente de hecho se apoya en las ayudas verbales. Mientras Lara toca simuladamente el violín, el profesor va haciendo preguntas sobre sus movimientos:

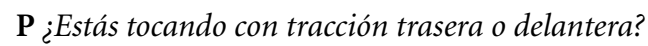

L Mmmmm

P Venga, compruébalo

L $\mathrm{Mmmm}$

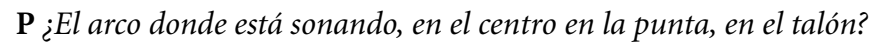

L En el centro

P Sí ahora está sonando en el centro, así que tracción trasera

P Canta lo que estás tocando, a la vez

L Sol, La

$\mathbf{P}$ Espalda del todo 
Las intervenciones docentes intentan focalizar la atención en la relación entre el cuerpo, la posición del arco y del violín, y tienen la función de ayudar a la alumna a explicitar verbalmente algunos componentes que están aún en parte implícitos en sus gestos (como acciones simuladas). Para ello, se utilizan diferentes representaciones externas: el gesto, el habla, cantar los sonidos que no se producen por la ausencia del violín.

Para que la alumna explicite mejor esos componentes implícitos en su simulación, el profesor graba la interpretación mediante su Smartphone mientras la estudiante toca la escala sin violín ni arco. Nada más finalizar la interpretación, ambos analizan los movimientos realizados, especialmente la coordinación entre esos movimientos (tracción trasera, tracción delantera) y las características de los sonidos alegres buscados (cortos, con ataque, con el arco sobre el centro...). El uso de esta grabación ayuda a tomar conciencia de los movimientos que se han realizado, de las dificultades y problemas con estos movimientos y son, por tanto, un primer paso para una futura autorregulación:

P Mira ¿qué ves aquí?

$\mathbf{L} \mathrm{Mmm}$

P Yo veo dos momentos, fijate... ¿Hasta aquí que pasa?

L Bien

$\mathbf{P}$ ¿Y después?

L Me voy más 'pallá' (reproduciendo con gestos el movimiento con el hombro y el brazo)

P Y eso ¿Por qué?

L Porque hago así y así (reproduciendo con gestos el movimiento)

P Estás en tracción trasera. Pim, pam, pum (cantando) y te pasas a la delantera

La alumna, guiada por las preguntas del profesor, intenta traducir esa representación externa (acciones corporales grabadas) a otros códigos (gestos, palabras) que le permiten establecer relaciones significativas entre los componentes de su interpretación (la emoción buscada, la relación con el instrumento imaginario y la posición o sensación corporal). Dado el carácter virtual de la interpretación, falta un último componente, el propio sonido. De hecho, tras este diálogo Lara toma el violín e interpreta la partitura de acuerdo con esas metas expresivas:

P Ahora, pam, pam, pam, (cantando) jAlegría!

(Lara comienza a tocar muy concentrada)

$\mathbf{P}$ Espalda atrás del todo (señala con la mano) recuerda que antes te pasabas a la delantera

Esto mismo se refleja en los WhatsApp que Lara envía después al profesor, tras ver las grabaciones que ha hecho en casa:

WhatsApp 10: [Tengo que] aumentar el sonido y expresividad y mantener la concentración en todas las notas que salen de mi cabeza//Y creo que no he sentido ni me he movido demasido porque a veces estoy demasiado concentrada en otras cosas. Y que no puedo pensar en tantas cosas a la vez, pero creoque lo he hecho bastante bien porque he pasado el arco recto y por cerca del puente y eso está muy bien yo creo. Tú qué piensas? 
En ellos parece que Lara ha interiorizado la relación entre esos componentes al valorar su propia interpretación. Esta evolución en la complejidad de su comprensión de la interpretación musical se refleja también en cómo va cambiando la regulación de sus acciones con el violín en función de las metas establecidas.

\section{Regulación metacognitiva de la interpretación musical}

Como hemos visto en el episodio de aula anterior, las interpretaciones de Lara van acompañadas de instrucciones verbales del profesor que llaman la atención sobre diferentes aspectos. La impresión que dan estas observaciones es que la regulación de la acción es externa. Parece que, al menos al comienzo, Lara necesita la ayuda de su profesor para regular sus acciones.

De hecho, en sus primeros WhatsApp queda claramente reflejada esta regulación externa, como vimos ya en un ejemplo anterior (WhatsApp 2: "En el primer video que te mandé la colocación de los dedos que me enseñaste en una parte no me acuerdo") en el que su autoevaluación intenta ajustarse a las demandas del docente. Igual sucede en este otro ejemplo:

WhatsApp 3: En este video te he enseñado lo de la espalda y el codo... Bueno, lo que me dijiste.

Pero, poco a poco, parece ir regulando mejor sus acciones, aunque estén todavía centradas en resultados técnicos, en la posición corporal con respecto al instrumento, sin considerar otros componentes y requieran la confirmación del docente.

WhatsApp 4: Opino que lo he hecho mejor porque he movido el brazo y no la mano como hacía antes

WhatsApp 6: Creo que he mejorado en lo de los cambios de posición y en el brazo.

Tu lo crees?. No lo se.)

Sigue necesitando de la ayuda del docente para hacer explícito el significado que estaba implícito en sus acciones y, como vimos antes, en sus gestos. Sabe si lo ha hecho bien o mal, aunque no es capaz de explicitar más allá de esas observaciones.

WhatsApp 8: Hola, creo que he dado más atención en el brazo y me he centrado en lo de los cambios de posición. El ritmo tengo que ensayarlo (-) ()) (i) Más con metrónomo.

En este último mensaje, además se puede observar cómo el uso de un objeto, el metrónomo, le permite también explicitar las dificultades relacionadas con un parámetro del sonido, el ritmo, así como usar ese objeto como un dispositivo representacional externo que le permite redescribir y regular su acción. Pero las evaluaciones conjuntas con su profesor le permiten ir un poco más allá de la reproducción de los resultados, regulando más sus acciones en función de otros componentes (emoción expresada, sonido, etc.). De hecho, en los últimos mensajes analiza algunos de los componentes que le han llevado a realizar bien o mal la tarea: 
WhatsApp 10: Y creo que no he sentido ni me he movido demasiado porque a veces estoy demasiado concentrada en otras cosas;

WhatsApp 11: Creo que lo he hecho bien porque he estado atenta a los cambios de cuerda y el vibrato. $Y$ he sentido lo que está en la obra

A pesar de estos avances, Lara sigue muy pendiente de los juicios del docente. Aunque es capaz de regular sus acciones con cierta autonomía, sigue asumiendo los criterios proporcionados por el docente en vez de construir los suyos propios:

WhatsApp 13: Creo que lo he hecho bastante bien porque he pasado el arco recto y por cerca del puente eso creo yo. Y también me he movido y tu me dijiste que si me movía lo hacía bien porque así es más expresivo y mola mucho más. Eso creo yo porque tu me dijiste que si me movía lo hacía bien y entonces me ha gustado mucho como lo he hecho.

Aunque Lara está comenzando a autorregularse, con grandes avances en los meses que lleva trabajando con este profesor, posiblemente por su edad y nivel de formación, y también por sus experiencias de aprendizaje previas, debe aún construir criterios de evaluación más autónomos.

En resumen, en esta clase de música hemos podido ver cómo el movimiento del cuerpo con una meta determinada, los gestos icónicos y parcialmente el instrumento pueden ser usados como representaciones externas que ayudan a explicitar los componentes y algunas de las posibles metas de la enseñanza, aunque esta explicitación está claramente mediada por la presencia de otra persona, el profesor que ayuda a dar significado a estas metas.

\section{Conclusiones}

A través del análisis de las secuencias de enseñanza y aprendizaje desarrolladas por Lara y su profesor, hemos podido ver que, desde un enfoque encarnado, el aprendizaje de la música se concibe como un proceso de redescripción de las propias experiencias y acciones corporales implicadas en la producción de sonidos con contenido emocional (Torrado, Pozo y Braga, 2016). El significado, o el sentido, de la interpretación musical es la comunicación emocional con el oyente. En este estudio nos interesaba ver el papel que han jugado diferentes tipos de representaciones externas entendidos como dispositivos semióticos, códigos o sistemas que permiten el diálogo entre representaciones de diferente naturaleza, en la construcción de ese conocimiento musical. También nos interesaba ver de qué modo el uso del Smartphone en el contexto del aula de música ayudaba a la explicitación de algunas de esas representaciones.

El punto de partida de las clases era la explicitación de los propios estados, sensaciones y movimientos corporales. Para ello se recurría a la propiocepción, haciendo que la alumna sintiera su cuerpo mientras intenta tocar, real o simuladamente, el violín. También se usaban grabaciones realizadas con el Smartphone para que la alumna relacionara esas representaciones internas, propioceptivas, con el visionado de las acciones realizadas y, en su caso, el sonido producido por su interpretación. El uso del Smartphone permitía focalizar 
la atención de la alumna en ciertos componentes motrices (la posición del brazo, la mano o la espalda), pero también expresivos (su expresión facial o su movimiento mientras tocaba). La relación entre esos diferentes planos de la representación corporal se gestionaba a través de un diálogo oral, cuando las acciones se realizaban en el aula, y también por medio de pequeños textos escritos y enviados por WhatsApp cuando la alumna ensayaba en casa y revisaba sus propias grabaciones. El uso de estos diferentes tipos de representaciones externas favorecía la recodificación o redescripción de las representaciones encarnadas primarias, dotándolas de nuevos y más complejos significados.

En muchas de las clases, incluida la que aquí hemos analizado, la alumna comenzaba tocando sin violín, utilizando representaciones miméticas que permitían concentrar toda su atención, y su explicitación, en esas sensaciones y movimientos corporales. Estos esbozos corporales, un tipo de gestos a los que Kirsh (2013) denomina "marking" tienen una clara función metarrepresentacional: sirven para codificar patrones de acción generalmente dirigidos a una meta expresiva concreta, en ausencia del instrumento. De hecho, tal como postula el propio Kirsh (2013), cuando mantiene que el marking es más eficaz para esa función que la propia realización efectiva de la acción, en alguna de las clases grabadas, pero no analizadas aquí por limitaciones de espacio, la alumna reconoce que en ese momento prefiere tocar sin violín porque cuando tiene el violín "se pone más tiesa y le sale peor" (Domínguez, 2016). Existen también otro tipo de gestos que se utilizan comúnmente en estas clases con fines representacionales explícitos. Como hemos mostrado con varios ejemplos, no es infrecuente que Lara, siendo aún incapaz de explicitar verbalmente sus ideas, responda a las preguntas del profesor mediante gestos, la mayor parte de ellos icónicos, pero también en algún caso metafóricos (Goldin-Meadow, 2004), mostrando así como estos gestos permitían una primera redescripción representacional, en formato E2 (o consciente no verbal) según Karmiloff-Smith (1992), todavía muy ligada a las representaciones implícitas y por tanto difícil de verbalizar o traducir a representaciones más simbólicas.

Hemos visto además que el propio instrumento, el violín y el arco, se convierten en dispositivos representacionales, que de algún modo representan las emociones y sonidos que se quieren transmitir ("con el arco así, alegría, toques cortos"). Los objetos sirven para explicitar las propias representaciones y para regular la propia acción, como cuando Lara recurre al metrónomo, un regulador externo, para ajustar el ritmo que aún no sabe regular internamente.

Pero si algo caracteriza a estas clases, y las diferencia de lo que podríamos llamar la enseñanza instrumental tradicional, es su naturaleza dialógica. En este tipo de clases, casi todas las acciones son reconstruidas a través del habla, no solo del profesor-como sucede en las clases tradicionales donde el profesor habla para ordenar, corregir, informar, etc. (López-Íñiguez y Pozo, 2016)-, sino también de la alumna. Las formas de hablar, como han mostrado los estudios ya clásicos sobre el análisis de las interacciones en el aula (Edwards y Mercer, 1987), son el medio esencial para construir y compartir significados. Lo interesante de las secuencias que hemos analizado es que ese habla remite siempre -tiene como contenido- a otros dispositivos representacionales, como el cuerpo, los gestos o los objetos, que con esta mediación verbal adquieren nuevos significados (tracción delantera, trasera, relacionado con la solemnidad o a alegría). 
Otro de los recursos empleados en estas clases es la escritura de WhatsApp, un código cercano al día a día de los alumnos, que sirve aquí como vehículo de conocimiento, con una clara función epistémica. La alumna debe tocar, experimentando todas las sensaciones propias de la interpretación, a continuación, observar en video lo que acaba de tocar y solo después intentar dar significado a lo que ha visto en función de las metas que tenía fijadas. El WhatsApp no cumple aquí una mera función pragmática, como ocurre cuando se usa en las redes sociales, sino que es un dispositivo representacional que, gracias a la pauta didáctica establecida por el profesor, promueve la reflexión sobre la propia acción. Además Lara incorpora a sus mensajes el carácter multimodal que caracteriza a este tipo de textos, usando emojis para explicitar los estados emocionales que acompañan a sus juicios y reflexiones.

Significativamente, el tipo de representación externa menos presente en estas clases es el que suele ocupar la mayor parte del tiempo en las aulas más tradicionales, la partitura, puesto que ya se ha hecho una representación interna de la misma. Eso no significa que no se trabaje -de hecho, Lara menciona en varias ocasiones la partitura cuando ensaya en casa-pero ocupa un papel menos central. Mientras toca, su atención no está plenamente puesta en la partitura sino en sus metas y en su propio cuerpo. La partitura es un recurso representacional más, pero no es el centro de la interpretación. Este peso limitado de la partitura y sus niveles de lectura se debe quizás también a que nos encontramos con una alumna que está aún en los niveles elementales del aprendizaje instrumental, , donde el repertorio es accesible a la representación del contenido expresivo y por su extensión y estructura, sencillo de recordar.

De hecho, desde esta propuesta se considera que el aprendizaje de los sistemas externos de representación musical, como las partituras, debe ayudar a reforzar y reconstruir la naturaleza expresiva de la música, que forma parte del bagaje cognitivo que todos los humanos, desde nuestra mente encarnada, compartimos. Todos, desde la infancia temprana, vivimos sumergidos en un entorno comunicacional, que incluye no solo el lenguaje natural, con la prosodia que lo acompaña, sino también la gestión de numerosos sonidos musicales con fines comunicacionales. Igual que la lectura y la escritura no son una mera traducción o extensión del habla, sino que la reconstruyen o redescriben generando nuevas formas de pensar y sentir, los sistemas externos de representación musical, aprendidos no solo en su nivel notacional o incluso sintáctico, sino sobre todo referencial o expresivo, pueden ayudar a redescribir esas experiencias musicales intuitivas. De hecho, sabemos que un enfoque complejo de la educación musical conlleva una forma distinta de aprender las partituras, más centrada en los niveles superiores que en la mera lectura nota a nota de las mismas.

No se trata por supuesto de abandonar la interpretación de los textos musicales, sino de entender que para que los alumnos se apropien de ellos - es decir, los hagan propios- necesitan disponer de recursos representacionales para traducir ese texto musical dentro de los componentes de su interpretación (las emociones que se desean comunicar, las acciones corporales, el sonido producido y el instrumento mediador). En este sentido, es importante destacar, como veíamos antes, que la presencia de representaciones simbólicas con mayor capacidad de abstracción o descontextualización no hace que se dejen de usar las representaciones más encarnadas, sino que unas se integran en otras, permitiendo tradu- 
cir a códigos simbólicos las representaciones más relacionados con el propio cuerpo, una traducción que requiere usar diferentes tipos de representaciones externas con fines epistémicos, es decir al servicio de la comprensión, el significado y la autonomía del intérprete.

\section{Agradecimientos}

Este trabajo es parte del Proyecto EDU2017-82243-C2-1-R financiado por el Ministerio de Ciencia, Innovación y Universidades de España.

\section{Notas}

1. Los mensajes están numerados según el orden en que han sido emitidos, no según el orden de presentación en este texto. Hemos respetado la grafía de Lara. Las erratas estaban en sus mensajes así como las barras que ha introducido para separar temas.

\section{Referencias}

Barab, S. A., y Dodge, T. (2008). Strategies for designing embodied curriculum. En: J.M. Spector, M.D. Merrill, J. Elen y M.J. Bishop. (Eds.) Handbook of research on educational communications and technology (pp. 97-110). N. York: Springer-Verlag.

Bautista, A., Pérez Echeverría, M.P. y Pozo, J.I. (2010). Music performance teachers' conceptions about learning and instruction: A Descriptive Study of Spanish Piano Teachers. Psychology of Music, 38, 85-106. DOI: 10.1177/0305735609336059

Bautista, A., Pérez Echeverría, M.P., Pozo, J.I. y Brizuela, B. (2009). Piano Students' Conceptions of Musical Scores as External Representations: A Transversal Study. Journal of Research in Music Education, 57(3), 181-202.

Casas-Mas, A. (2016). Mediaciones instrumentales entre distintas culturas de aprendizaje musical. Mediaciones sociales (15), 151-167.

Casas-Mas, A., López-Íñiguez, G., Pozo, J.I. y Montero, I. (2018). The Function of Private Singing in Instrumental Music Learning: A Multiple Case Study of Self-regulation and Embodiment. Musicae Scientiae. DOI: 10.1177/1029864918759593

Casas-Mas, A., Pozo, J.I. y Scheuer, N. (2015). Musical Learning and Teaching Conceptions as Sociocultural Productions in Classical, Flamenco and Jazz Cultures. Journal of CrossCultural Psychology, 46 (9), 1191-1225.

Claxton, G. (2015). Intelligence in the Flesh: Why Your Mind Needs Your Body Much More Than it Thinks. Londres: Yale University Press.

Cook, N. (2013). Beyond the score: Music as performance. Oxford, Oxford University Press.

Cox, A. (2016). Music and embodied cognition: Listening, moving, feeling, and thinking. Indiana: Indiana University Press.

Damasio, A. (1994). Descartes's error. Emotion, reason and the human brain. N. York: Avon Books. 
Davies, S. (1994). Musical Meaning and Expression. N. York: Cornell University Press.

De Vega, M., Glenberg, A. M. y Graesser, A.C. (Eds.) (2008). Symbols and embodiment. Debates on meaning and cognition. Oxford: Oxford University Press.

Domínguez, A. (2016). El Cuerpo como Mediación Representacional en el Aprendizaje de la Música. Trabajo de Fin de Grado. Faulted de Psicología. Universidad Autónoma de Madrid.Donald, M. (1991). Origins of the modern mind. Three stages in the evolution of culture and cognition. Cambridge, MA: Harvard University Press.

Edwards, D. y Mercer, N. (1987). Common knowledge. The development of understanding in the classroom. Londres: Methuen and Co. [Trad. Cast.: El conocimiento compartido. El desarrollo de la comprensión en el aula. Barcelona: Paidós-MEC, 1998].

Gabrielsson, A., y Lindström, E. (2010). The role of structure in the musical expression of emotions. En P. N. Juslin y J. A. Sloboda (Eds.), Handbook of music and emotion: Theory, research, applications (pp. 367-400). N.York: Oxford University Press.

Glenberg, A. S., De Vega, M. y Graesser, A.C (2008). Framing the debate. En: M. de Vega; A. M. Glenberg y A. C. Graesser (Eds.) Symbols and embodiment. Debates on meaning and cognition (pp. 1-9). Oxford: Oxford University Press.

Goldin-Meadow, S. (2003). Hearing gesture: How our hands help us think. Cambridge, Mass.: Harvard University Press.

Juslin, P. N., y Västfjäll, D. (2008). Emotional responses to music: the need to consider underlying mechanisms. The Behavioral and Brain Sciences, 31(5), 559-575; discussion 575-621. doi:10.1017/S0140525X08005293.

Karmiloff-Smith, A. (1992). Beyond modularity. Cambridge, Mass.: Cambridge University Press. [Trad. cast.: Más allá de la modularidad de la mente: la ciencia cognitiva desde la perspectiva del desarrollo. Madrid: Alianza Editorial, 1995].

Kirsh, D. (2013). Embodied cognition and the magical future of interaction design. ACM Transactions on Computer-Human Interaction, 20 (1), 3-30.

Kivy, P. (2001). New essays on musical understanding. Oxford: Oxford University Press.

Koelsch S. (2014). Brain correlates of music-evoked emotions. Nature Reviews. Neuroscience, 15, 170-180. https://www.pubchase.com/article/24552785.

Koelsch, S., Kilches, S., Steinbeis, N., y Schelinski, S. (2008). Effects of unexpected chords and of performer's expression on brain responses and electrodermal activity. PLoS One, 3 (7), e2631.

Koopman, C., y Davies, S. (2001). Musical meaning in a broader perspective. The Journal of Aesthetics and Art Criticism, 59 (3), 261-273.

López-Íñiguez, G. y Pozo, J.I. (2014). The influence of teachers' conceptions on their students' learning: children's understanding of sheet music. British Journal of Educational Psychology, 84(2), 311-328. doi: 10.1111/bjep.12026

López-Î́niguez, G. y Pozo, J.I. (2016). Analysis of constructive practice in instrumental music education: Case study with an expert cello teacher. Teaching and Teacher Education, 60, 97-107. doi:1016/j.tate.2016.08.002

Marín, C., Pérez Echeverría, M. P. y Hallam, S. (2012). Using the musical score to perform: A study with Spanish flute students. British Journal of Music Education, 29(2), 193-212. doi: $10.1017 /$ S0265051712000046 
Marín, C., Scheuer, N. y Pérez-Echeverría, M. P. (2013). Formal music education not only enhances musical skills, but also conceptions of teaching and learning: a study with woodwind students. European Journal of Psychology of Education, 28(3), 781-805. 10.1007/ s10212-012-0140-7

Martí, E. (2003). Representar el mundo externamente. La adquisición infantil de los sistemas externos de representación. Madrid: Antonio Machado.

Perdomo-Guevara, E. (2014). Is music performance anxiety just an individual problem? Exploring the impact of musical environments on performers' approaches to performance and emotions. Psychomusicology: Music, mind, and brain, 24 (1), 66.

Pérez Echeverría, M. P., Martí, E. y Pozo, J. I. (2010). Los sistemas externos de representación como herramientas de la mente. Cultura y Educación, 22(2), 133-147.

Pérez Echeverria, M.P; Postigo, Y. y Marín, C. (2018). Understanding of graphs in social science undergraduate students: selection and interpretation of graphs, Irish Educational Studies DOI 10.1080/03323315.2018.1440248

Pérez Echeverría, M.P. y Scheuer, N. (2009). External representations as learning tools. An introduction. En: C. Andersen; N. Scheuer, M.P.; Pérez Echeverría y E. Teubal (Eds.): Representational Systems and Practices as Learning Tools in different Fields of Learning (págs. 1-18). Rotterdam: Sense.

Postigo, Y. y Pozo, J.I. (2004). On the road to graphicacy: the learning of graphical representation systems. Educational Psychology, 24 (5), 623-644.

Pozo, J.I. (2001). Humana mente: el mundo, la conciencia y la carne. Madrid: Morata.

Pozo, J.I. (2008). Aprendices y maestros: la psicología cognitiva del aprendizaje. Segunda Edición. Madrid: Alianza.

Pozo, J.I. (2014). Psicología del Aprendizaje Humano: adquisición de conocimiento y cambio personal. Madrid: Morata.

Pozo, J.I. (2017). Learning beyond the body: from embodied representations to explicitation mediated by external representations. Infancia y Aprendizaje, 40 (2), 219-276, DOI: 10.1080/02103702.2017.1306942.

Rivière, A. (2003). Teoría de la mente y metarrepresentación. En: A. Rivière: Obras escogidas. Vol I. (págs. 191-231). Madrid: Ed. Médica Panamericana.

Rowlands, M. (2010). The new science of the mind. Cambridge, Mass.: The MIT Press.

Tomasello, M. (1999). The cultural origins of human cognition. Cambridge, Mass.: Harvard University Press.

Torrado, J.A., Jiménez, P: Rucandio, I. y Pozo, J.I. (2017). Aprendiendo a enseñar música: un nuevo enfoque para la formación de profesores. I Congreso Internacional de Psicología de la Música y la interpretación musical. Madrid: UNED.

Torrado, J.A., Pozo, J.I. y Braga; M. (2016). Expresividad: un motor para el aprendizaje de la técnica. IV Congreso CEIMUS de. Educación e Investigación musical: las experiencia creativa en la pedagogía de la música. Torrejón de Ardoz: Enclave Creativa Ediciones S.L.

Vygotski, L.S. (1978). Mind and society. The development of higher psychological processes. Cambridge, Mass.: Harvard University Press.

Wilson, M. (2002). Six views of embodied cognition, Psychonomic Bulletin and Review, 9, 625-636. 


\begin{abstract}
This paper presents a single case, descriptive study in music teaching. Instead of following the more analytic, traditional teaching methods, based on the processing of musical scores, this study assumes an emotional and embodied approach to music learning. In order to uncover the processes of representational redescription needed to make sense of instrumental music, we analyze the specific role played by different representations, from the embodied representations, including gestures or body positions and movements, all the way to the use of symbolic systems, such as the scores. We also study how the use of Smartphones in the classroom can contribute to an epistemic approach to music learning and teaching. Finally, we contrast these different teaching models and suggest a functional integration of different kind of representations in music classrooms.
\end{abstract}

Keywords: emotions - embodied representations - symbolic representations - learning of music - representational redescription.

Resumo: Este artigo apresenta um trabalho descritivo de ensino de música, baseado em um caso, no qual, a partir de uma abordagem emocional e encarnada, longe dos métodos tradicionais, analítica e baseada na partitura, mostra o papel desempenhado por diferentes representações, do mais encarnado (posição corporal, gestos) ao mais simbólico (a partitura), no processo de redescrição representacional para dar um significado emocional à música. Além disso, estuda-se como o uso do Smartphone e outros dispositivos similares podem auxiliar uma abordagem epistêmica ao ensino e aprendizado da música. As diferentes abordagens abordadas na música e as relações entre diferentes representações são discutidas.

Palabras chave: Emoções - representações corporificadas - representações simbólicas aprendizado de música - redescrição representacional.

[Las traducciones de los abstracts fueron supervisadas por el autor de cada artículo] 